\title{
Numerical probability analysis of low-temperature insulation destruction under the condition of periodic duty
}

\author{
V.Yu. Polovnikov and M.V. Piskunov \\ Tomsk Polytechnic University, 30, Lenin Avenue, Tomsk 634050, Russia
}

\begin{abstract}
The numerical investigation of thermal stresses within low-temperature insulation covering cryogenic pipelines and the numerical probability analysis of lowtemperature insulation destruction under the condition of periodic duty were carried out. The minimal longevity values for foamed polyurethane and mineral cotton were established. The results of longevity analysis for foamed polyurethane and mineral cotton under the condition of environment temperature variation were obtained.
\end{abstract}

\section{Introduction}

It is important and necessary to develop the insulation materials which exhibit good mechanical strength and radiation resistance at cryogenic temperatures [1].

A common property of all cryogenic insulation systems is that they have to be protected against penetration of water steam, because of their low service temperature level, otherwise their efficiency is strongly reduced [2].

Thermal insulation of cryogenic piping transporting liquefied natural gas at a temperature of $-160^{\circ} \mathrm{C}$ is operated under heavy conditions, and first of all it is a continuous variation of temperature and outer air humidity.

There are some investigations [1, 3, 4] concerning problems associated with the mechanical properties, strength and thermoelasticity. It is interesting to note that nowadays there are no such things as model and analysis procedure of usage frequency of cryogenic facilities. Therefore, the development of approaches regarding the longevity evaluation of insulating materials and the service life prediction of low-temperature insulation taking into account the usage frequency of pipelines obtain the special relevance.

The purpose of this study is numerical investigation of thermal stresses within low-temperature insulation covering cryogenic pipelines and the probability analysis of insulation destruction under the condition of periodic duty.

\section{Problem statement}

The cryogenic pipeline section transporting the liquefied natural gas (LNG) at a temperature of $\approx-161^{\circ} \mathrm{C}$ is considered. The low-temperature pipeline is covered with insulation (foamed 


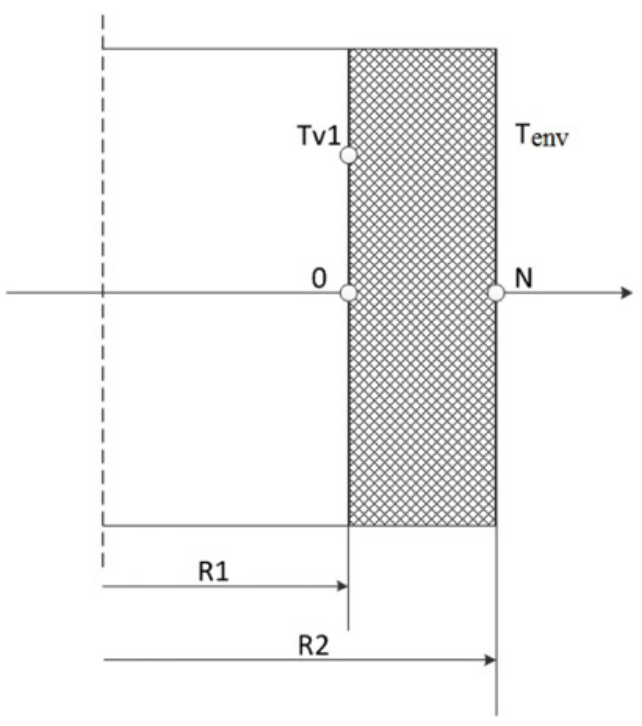

Figure 1. A scheme of decision domain: R1 - pipeline radius; (R2 - R1) - insulation thickness; Tenv - environment temperature; Tv1 - temperature of cryogenic liquid.

polyurethane, mineral cotton). The thermal stresses arise inside the insulation due to cooling. The unidimensional non-stationary problems of thermal conductivity and thermoelasticity and also the longevity evaluation of insulating materials according to kinetic durability conception of solid-state bodies are conjointly resolved.

The outer duct of pipe coat contacts environment. Regarding the heat conduction problem, the firsttype and third-type boundary conditions were introduced on the internal and external boundaries of heat-insulating layer, respectively. The temperature equals the constant value at the initial instant. It is estimated that inside temperature of insulation equals temperature of cryogenic fluid $T_{v 1}$. Moreover, stresses due to temperature arise from insulation cooling. The heat transfer inside the insulation takes place only by using thermal conductivity.

\section{Mathematical model}

Mathematical statement of problem is described by the following equations:

$$
\frac{\partial T}{\partial t}=a\left(\frac{\partial^{2} T}{\partial r^{2}}+\frac{1}{r} \frac{\partial T}{\partial r}\right)
$$

Most models found in the literature combine solid and gas thermal conductivity using geometric simplification of the internal structure of the foam [5-7].

It is considered that the temperature is varied only within the insulation thickness $T=T(r)$ :

$$
\begin{gathered}
\sigma_{y y}=\sigma_{z z}=\sigma(r), \sigma_{x x}=\sigma_{x z}=\sigma_{x y}=0 \\
\sigma(r, \tau)=\frac{\alpha \cdot E}{1-v}\left[\frac{1}{\delta} \int_{0}^{\delta} T(r, \tau) d x-T(r, \tau)\right] .
\end{gathered}
$$


Table 1. Thermal and physical characteristics for insulating materials.

\begin{tabular}{|l|l|l|l|l|l|l|l|}
\hline Insulating materials & $\begin{array}{c}\boldsymbol{k}, \\
{[\mathbf{W} /(\mathbf{m} \cdot \mathbf{K})]}\end{array}$ & $\begin{array}{c}\boldsymbol{c}, \\
{[\mathbf{k J} /(\mathbf{k g} \cdot \mathbf{K})]}\end{array}$ & $\begin{array}{c}\boldsymbol{\rho}, \\
{\left[\mathbf{k g} / \mathbf{m}^{\mathbf{3}}\right]}\end{array}$ & $\begin{array}{l}\boldsymbol{\alpha}_{\mathbf{p}} \cdot \mathbf{1 0}^{-\mathbf{6}}, \\
{\left[\mathbf{K}^{-\mathbf{1}}\right]}\end{array}$ & $\begin{array}{c}\boldsymbol{E}, \\
{[\mathbf{M P a}]}\end{array}$ & $\begin{array}{c}\boldsymbol{\boldsymbol { \sigma }}, \\
{[\mathbf{M P a}]}\end{array}$ \\
\hline Foamed polyurethane & 0.0397 & $0.46-0.8$ & 390 & $66-71$ & 1.15 & 0.33 & 1.4 \\
\hline Mineral cotton & 0.047 & 0.88 & 130 & 7.7 & $0.15-0.25$ & 0.17 & $0.01-0.04$ \\
\hline
\end{tabular}

Table 2. Results of longevity numerical analysis for foamed polyurethane.

\begin{tabular}{|c|c|}
\hline Conditions & $\tau(\boldsymbol{\sigma})$, year \\
\hline At $T_{\text {average }}=215.5 \mathrm{~K}$ and $\sigma_{\max }=1.78 \cdot 10^{4} \mathrm{~Pa}$ & 31 \\
\hline At $T_{\text {average }}=215.5 \mathrm{~K}$ and $\sigma_{\text {stab }}=1.65 \cdot 10^{4} \mathrm{~Pa}$ & 31.33 \\
\hline
\end{tabular}

The Equation of longevity evaluation:

$$
\tau_{\partial}=\tau_{0} \exp \frac{U_{0}-\gamma \sigma}{k T_{\text {average }}} .
$$

The initial conditions:

$$
T=T_{\text {init }}=\text { const }, \quad R_{1} \leq r \leq R_{2}, \quad \tau=0 ;
$$

The boundary conditions:

$$
\begin{array}{r}
r=R_{1}, T=T_{v 1}=\text { const }, \tau>0 ; \\
-k \frac{\partial T}{\partial r}=\alpha\left(T-T_{\text {env }}\right), \quad r=R_{2}, \quad \tau>0 ;
\end{array}
$$

\section{Method of solution and initial data}

The problem (1)-(6) was solved by using Thomas algorithm [8], Simpson method and by using formula by S.N. Zhurkova (member of the science academy).

The typical cryogenic pipeline section in diameter $400 \mathrm{~mm}$ was considered. The mineral cotton and foamed polyurethane thermal insulation (closed-cell polyurethane foam) in thickness $280 \mathrm{~mm}$ were selected as insulating material. The inner boundary temperature $R_{1}$ equals temperature of cryogenic fluid $T_{v 1} \approx-161^{\circ} \mathrm{C}$. The maximal environment temperature is $T_{e n v}=45^{\circ} \mathrm{C}$. The heat-exchange coefficient from air to insulation surface is $\alpha=5 \mathrm{~W} /\left(\mathrm{m}^{2 \cdot{ }^{\circ}} \mathrm{C}\right)$. The action duration time of thermal stresses was equal to $\tau=14 \mathrm{~h}$.

Thermal and physical characteristics for materials used under numerical simulation are listed in the Table 1.

\section{Results of numerical simulation}

The main numerical simulation results of thermal stresses within low-temperature insulation covering cryogenic pipelines are shown in Figs. 2 and 3.

The main results of longevity numerical analysis for foamed polyurethane and mineral cotton are shown in Tables 2 and 3.

The obtained results of longevity analysis for foamed polyurethane have the normal values taking into account the design data of low-temperature pipelines used in Sakhalin-2 project. The lifetime of low-temperature pipelines is 30 years and more. The parameter of moisture absorption and the potential freezing of insulating layer are necessary to take into account for adequacy of results for mineral cotton. 

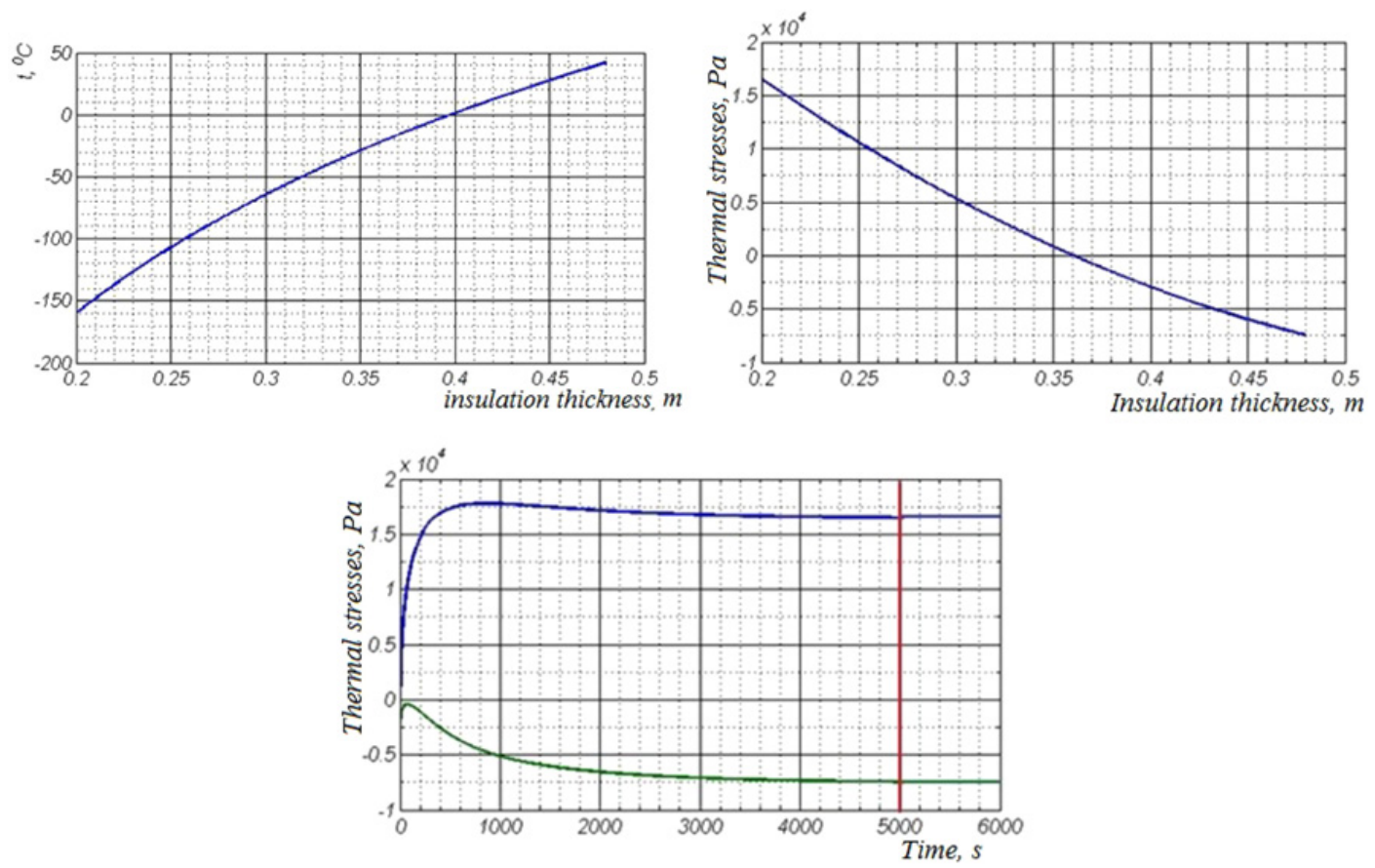

Figure 2. Simulation results for foamed polyurethane (Red line - starting time of stabilization of thermal stresses values).
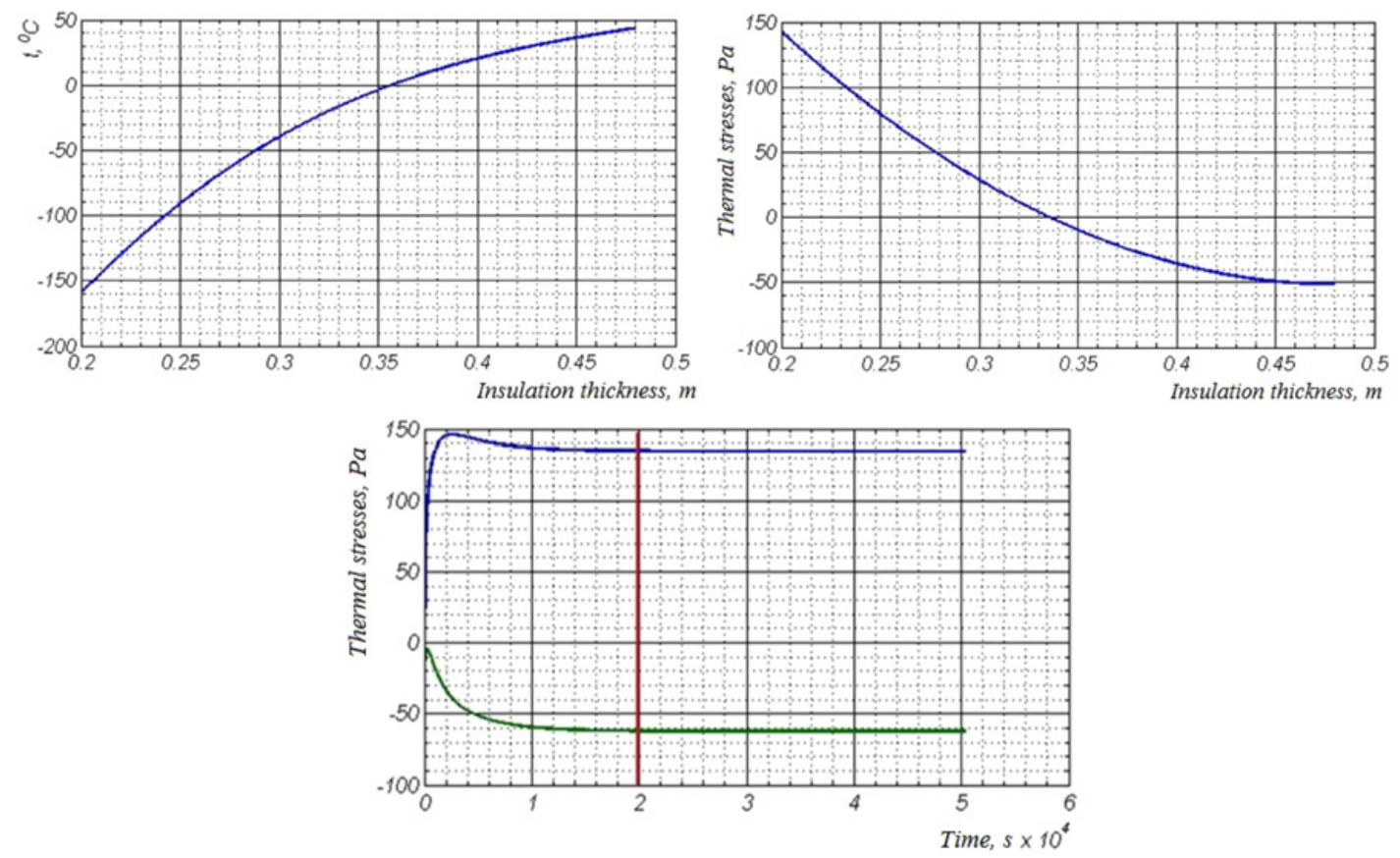

Figure 3. Simulation results for mineral cotton (Red line - starting time of stabilization of thermal stresses values). 
Table 3. Results of longevity numerical analysis for mineral cotton.

\begin{tabular}{|c|c|}
\hline Conditions & $\tau(\boldsymbol{\sigma})$, year \\
\hline At $T_{\text {average }}=215.5 \mathrm{~K}$ and $\sigma_{\max }=146 \mathrm{~Pa}$ & $8.9 \cdot 10^{14}$ \\
\hline At $T_{\text {average }}=215.5 \mathrm{~K}$ and $\sigma_{\text {stab }}=135 \mathrm{~Pa}$ & $8.9 \cdot 10^{14}$ \\
\hline
\end{tabular}

Table 4. Results of longevity analysis for foamed polyurethane under the condition of environment temperature variation.

\begin{tabular}{|c|c|c|c|c|c|c|}
\hline $\begin{array}{l}\text { Environment } \\
\text { temperature } \mathbf{T}, \mathbf{K}\end{array}$ & $\boldsymbol{\sigma}_{\max }, \mathbf{P a} \cdot \mathbf{1 0}^{\mathbf{4}}$ & $\boldsymbol{\tau}_{\text {load }}, \mathbf{s .}$ & $\boldsymbol{\sigma}_{\text {stab}}, \mathbf{P a} \cdot \mathbf{1 0}^{\mathbf{4}}$ & $\boldsymbol{\tau}_{\text {load }}, \mathbf{s .}$ & $\tau\left(\boldsymbol{\sigma}_{\text {max }}\right)$, year & $\tau\left(\boldsymbol{\sigma}_{\text {stab }}\right)$, year \\
\hline 308 & 1.74 & 845 & 1.62 & 5000 & 127.40 & 128.30 \\
\hline 298 & 1.70 & 851 & 1.59 & 4240 & 476.10 & 479.60 \\
\hline 283 & 1.64 & 839 & 1.54 & 4324 & $3.90 \cdot 10^{3}$ & $3.93 \cdot 10^{3}$ \\
\hline 263 & 1.57 & 851 & 1.47 & 4260 & $84.20 \cdot 10^{3}$ & $85 \cdot 10^{3}$ \\
\hline $\begin{array}{l}275.8 \text { (average annual } \\
\text { temperature in Yuzno) }\end{array}$ & 1.61 & 851 & 1.51 & 4536 & $83 \cdot 10^{3}$ & $83.8 \cdot 10^{3}$ \\
\hline
\end{tabular}

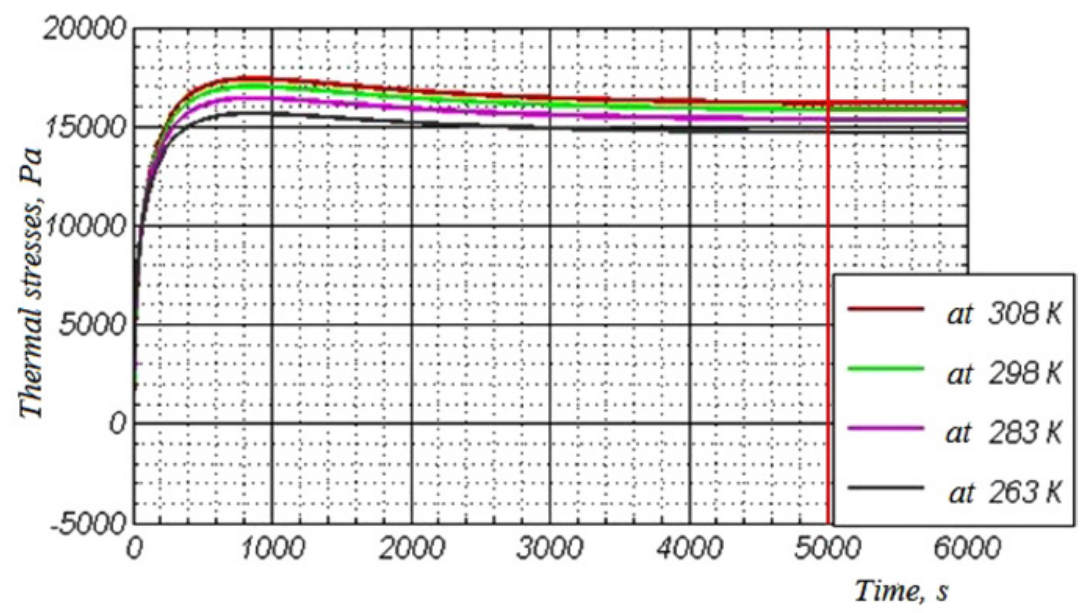

Figure 4. Distribution of thermal stresses on the inside surface of foamed polyurethane depending on loading time under the condition of various environment temperature (Red line - starting time of stabilization of thermal stresses values).

It is necessary to point out that, as expected, the increase of longevity value under the condition of decrease of thermal stresses values (under the condition of constant average temperature along the insulation thickness) is observed.

The results of longevity analysis for foamed polyurethane under the condition of environment temperature variation are listed in Table 4.

The distribution of thermal stresses on the inside surface of foamed polyurethane depending on loading time under the condition of various environment temperature is shown in Fig. 4.

An analysis of the results listed in Fig. 4 allows to draw the main conclusion that when increasing environment temperature, thermal stresses values increase. Moreover, when increasing the environment temperature (Table 4), the reducing of lifetime for insulating material is observed.

The results of longevity analysis for mineral cotton under the condition of environment temperature variation are listed in Table 5. 
Table 5. Results of longevity analysis for mineral cotton under the condition of environment temperature variation.

\begin{tabular}{|c|c|c|c|c|c|c|}
\hline $\begin{array}{l}\text { Environment } \\
\text { temperature } T, \mathbf{K}\end{array}$ & $\boldsymbol{\sigma}_{\text {max }}, \mathbf{P a}$ & $\tau_{\text {load }}, \mathbf{S .}$ & $\boldsymbol{\sigma}_{\text {stab }}, \mathbf{P a} \cdot \mathbf{1 0}^{\mathbf{4}}$ & $\tau_{\text {load }}, \mathbf{S .}$ & $\tau\left(\boldsymbol{\sigma}_{\max }\right)$, year & $\tau\left(\boldsymbol{\sigma}_{\text {stab }}\right)$, year \\
\hline 308 & 152.30 & 1652 & 141 & 12043 & $7.08 \cdot 10^{13}$ & $7.08 \cdot 10^{13}$ \\
\hline 283 & 143.80 & 1654 & 134 & 11450 & $1.22 \cdot 10^{16}$ & $1.22 \cdot 10^{16}$ \\
\hline 263 & 137 & 1652 & 128.5 & 10205 & $1.24 \cdot 10^{18}$ & $1.24 \cdot 10^{18}$ \\
\hline
\end{tabular}

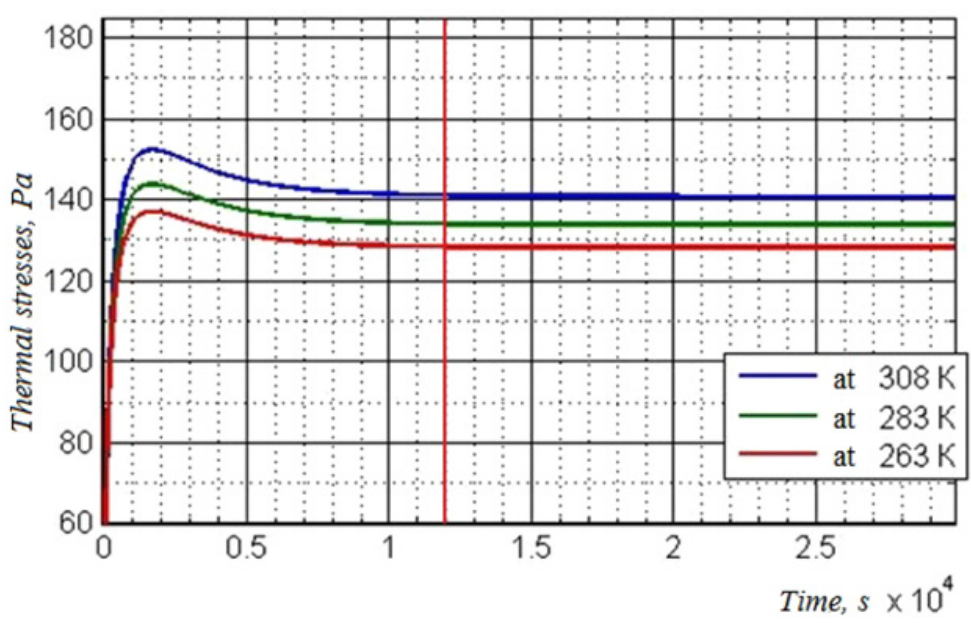

Figure 5. Distribution of thermal stresses on the inside surface of mineral cotton depending on loading time under the condition of various environment temperature (Red line - starting time of stabilization of thermal stresses values).

The distribution of thermal stresses on the inside surface of mineral cotton depending on loading time under the condition of various environment temperature is shown in Fig. 5.

The simulation results for mineral cotton shown in Table 5 and Fig. 5 allow to make the analogous conclusions as for foamed polyurethane.

\section{Conclusion}

The numerical investigation of thermal stresses within low-temperature insulation covering cryogenic pipelines and the numerical probability analysis of low-temperature insulation destruction under the condition of periodic duty were carried out. It has been established that minimal longevity value for foamed polyurethane is 31 years, for mineral cotton is $8.9 \cdot 10^{14}$ years. The results of longevity analysis for foamed polyurethane and mineral cotton under the condition of environment temperature variation were obtained.

This work was supported by the Grants Council (under RF President), grant No. MK-1652.2013.8. and by the Russian Fond of Basic Research, grant No. 12-08-00201. 


\section{Notations}

$R$ - boundary of computational domain; $T$ - temperature, $\mathrm{K} ; a$ - temperature conductivity coefficient, $\mathrm{m}^{2} / \mathrm{s} ; \tau$ - time, $\mathrm{s} ; r$ - coordinate position, $\mathrm{m} ; k$ - heat conduction coefficient, $\mathrm{W} /(\mathrm{m} \cdot \mathrm{K}) ; \alpha$ - heat-exchange coefficient, $\mathrm{W} /\left(\mathrm{m}^{2} \cdot \mathrm{K}\right) ; \sigma$ - component of a stress; $v$ - the Poisson ratio; $E$ - modulus of elasticity, $\mathrm{MPa}$; $\lambda$ and $\mu$ - Lame constants; $\varepsilon$ - strain component; $e$ - volume strain; $\alpha_{\mathrm{p}}$ - linear thermal expansion coefficient, $\mathrm{K}^{-1} ; \tau_{0}=10^{-14} \mathrm{~s}$. - period of thermal oscillation of atoms (for polymeric compounds); $U_{0}$ - activation energy of destruction process, $\mathrm{J} / \mathrm{mole} ; k$ - Boltzmann constant, $\mathrm{J} / \mathrm{K} ; T_{\text {average }}$ - average insulation temperature along the thickness, $\mathrm{K} ; \gamma$ - structure-sensitive coefficient, $\mathrm{m}^{3} ; \tau(\sigma)$ - longevity of insulating material, year; $\sigma_{\max }-$ maximum of thermal stresses, Pa; $\sigma_{\text {stab }}-$ stabilized value of thermal stresses, Pa.

Indices: init - the initial instant; 1,2 - numbers of area borders of calculation; env - environment; $v 1$ - cryoliquid.

\section{References}

[1] Zhixiong Wu, Hao Zhang, Huihui Yang, Xinxin Chu, Yuntao Song, Weiyue Wu, Huajun Liu, Laifeng Li, Properties of radiation stable, low viscosity impregnating resin for cryogenic insulation system, Cryogenics. 51 (2011) 229-233

[2] A. Hofmann, The thermal conductivity of cryogenic insulation materials and its temperature dependence, Cryogenics. 46 (2006) 815-824

[3] V.E. Gul, Influence of Change of Relaxation Properties on Polymer Tensile Strength, International Journal of Polymeric Materials and Polymeric Biomaterials. 20 (1993) 165-179

[4] Wei-Ting Lin, An Cheng, Ran Huang, Tsan-Ching Cheng, A method for testing the strength of concrete using uniaxial direct tension, Journal of the Chinese Institute of Engineers. 36 (2013) 295-303

[5] M. Barrios, S.W, Van Sciver, Thermal conductivity of rigid foam insulations for aerospace vehicles, Cryogenics. 55-56 (2013) 12-19

[6] Glicksman, Heat transfer in foams. In: Hilyard NC, Cunningham A, editors. Low density thermal plastics. London: Chapman and Hall, 1994

[7] M.A. Schuetz, L.R. Glicksman, A basic study of heat transfer through foam insulation, J Cell Plast. 20 (1987) 114-135

[8] A.L. Garcia, Numerical methods for physics, Prentice Hall, New York, 2000 